\title{
Research With Tribal Communities and Peoples: Progress, Challenges and Looking to the Future
}

\author{
Martha W. Waller ${ }^{1}$
}

Published online: 3 February 2017

(C) Springer Science+Business Media New York 2017

\section{Introduction}

The decision of the editorial team of the Journal of Primary Prevention to develop this special double issue came about while we were in a meeting debating whether a recently submitted manuscript should go out for review. In these weekly meetings, our team considers the quality of the research of each newly submitted manuscript as well as the relevance and appeal of its topic to our many audiences. When it comes to high quality prevention research with diverse ethnic populations, there is a definite dearth and this is particularly true of studies relating to Native American populations. There are many legitimate (and probably some non-legitimate) reasons for this, of course, but it means that prevention providers and public health professionals working with Tribal communities often lack strong scientific evidence to support or inform their work, and thus find themselves without the means to guide, justify and support their decisions.

Conducting successful research with Native peoples, and within Tribal community settings, requires Tribal peoples' meaningful involvement and great sensitivity to Tribal culture, history, tradition, and cultural protocols. It takes considerable time to develop relationships and trust in Native communities and often requires a community-based participatory research (CBPR) approach to be successful. Both the time involved and the participatory nature of the work can discourage many investigators who may be interested in pursuing research efforts with Tribal communities, because the practicalities involved in the development of effective

Martha W. Waller

mwaller@PIRE.org

1 Pacific Institute for Research and Evaluation (PIRE), 101 Conner Drive, Suite 200, Chapel Hill, NC 27514-7038, USA 
collaborations are not typically supported by funders. Yet despite these challenges, the authors of the manuscripts in this issue have made great inroads in documenting the nuances of health and well-being among Native peoples, in a manner that is fully respectful of the participants.

We are pleased to bring you nine original research manuscripts that address such topics as mental health and cultural connectedness, the mentoring of Native youth, sexual health curricula targeting Native youth, stress among Native women, smoking behaviors on and off Tribal lands, and patient navigator programs for Natives with cancer. These studies employ both qualitative and quantitative methods that are designed to reveal, understand, and promote the health and wellbeing of Native adults and youth. In addition, we have included a literature review of protective factors and health among Native American youth and a brief report on the development of a child safety curriculum targeting Native American families. Of particular note is the emphasis by many of the studies on exploring, incorporating and utilizing the inherent strengths of Native cultures and peoples to improve health by understanding how ties to family, spirituality, culture, and mentors are associated. Henson and colleagues reviewed the literature to identify protective factors identified specifically among Native American and Alaskan Native youth; Snowshoe and colleagues found that cultural connectedness was strongly associated with life satisfaction among First Nations youth; and Kulis, Shegog, Grimes, Usera, and Berns and their respective colleagues each developed culturally focused prevention programming or adapted existing programs to be more culturally informed.

The research in this issue moves our understanding of Native American health forward, while also highlighting how much work remains. Western researchers still understand very little about the effects of historical trauma on health, whether healing from historical trauma can be achieved, what it might look like, and how it might be achieved. We are just starting to explore how culture and spirituality can strengthen the resiliency of families and youth, and we know even less about whether and how lateral violence in Native communities may discourage progress and affect health, and how it might be addressed. Finally, because the tendency among researchers to assume homogeneity among Native peoples is also inherent in many research perspectives, studies that explore the heterogeneity that exists would also be an important focus for future research.

Native communities, like every community, often face numerous and unique challenges. Within these communities there are elders, leaders, educators, providers, and others who have the desire, will, and ability to create change from within. When Native communities are broached with research that aligns with their values and are true partners, transformative change can be achieved. CBPR is one approach that builds on a community's strengths, values, culture, and needs, while also resulting in strong, high quality research for which the community can rightly take responsibility. But, as previously mentioned, this approach also requires a commitment on the part of the community and researchers, not to mention the funders, that may be challenging and that few are able to fully accomplish. In addition, the way time is viewed by the dominant society and Tribal communities often is incongruent, which further complicates the ability of investigators to use 
research methods that are in line with Native communities' world views. This may partly explain why we still find that the field of research with Native American and aboriginal communities, and our understanding of protective and risk factors related to health among Native peoples, remain less developed than those with majority populations.

We would like to thank the many reviewers of the manuscripts (and of this introduction as well) who provided thoughtful and critical feedback on the studies included in this special issue. We hope that this issue will inspire questions, discussions, and new research methods and ideas. 